\title{
RISK AND HAZARD ASSESSMENT OF EXTREME NATURAL EVENTS FOR CRITICAL INFRASTRUCTURES
}

\author{
J.-U. KLÜGEL \\ NPP Goesgen-Daeniken, Switzerland.
}

\begin{abstract}
Human society is frequently surprised by extreme natural events that lead to tremendous losses both in human lives as well as in economic capital. Risk assessment methods regularly fail to predict such disasters by treating extreme events as low probability, tolerable ones. Critical infrastructures and life lines are often found to be poorly protected due to their inadequate design basis. A method of hazard assessment for extreme natural events is presented that allows for the consideration of unexpected extreme events ('black swan' events). Including such events into the design basis prevents the failure of critical infrastructures due to 'cliff-edge' effects. The method makes use of some general properties of heavy-tailed distributions and the mathematical theory of records, and takes advantage of a distribution-free approach without need to calculate probabilities of exceedance. The application of the method is demonstrated on several examples for nuclear power plants (NPP), including High Wind and High Temperature hazards. The results are compared with the results of hazard assessment using conventional probabilistic hazard analysis methods. The risk implications are discussed. Keywords: black swan theory, critical infrastructures, natural hazards, risk assessment.
\end{abstract}

\section{INTRODUCTION}

Human society is frequently surprised by extreme natural events that lead to tremendous losses both in human lives as well as in economic capital. Among such events there has to be listed the Nicobar-Andaman earthquake with the subsequent tsunami (2004), the hurricane Katrina or the Big Japan Earthquake of 2011 which led to the destruction of the nuclear power plant (NPP) of Fukushima Daiichi as a consequence of the complete loss of internal and external infrastructure supporting the plant's safety systems [1]. In these cases, traditional probabilistic hazard assessment methods and their linked decision criteria (e.g. using a probability of exceedance of $10^{-4}$ for the selection of the design basis hazard) failed to identify the possibility of occurrence of very extreme events during the lifetime of critical infrastructures. The accident of Fukushima and the identified limitations of traditional probabilistic hazard assessment methods have led to the decision of Western European Nuclear Regulatoy Authorities (WENRA) to support an integrated approach to hazard assessment giving preference to deterministic methods. WENRA recommends that (quotation), 'For all natural hazards that have not been screened out, hazard assessments shall be performed using deterministic and, as far as practicable, probabilistic methods taking into account the current state of science and technology'. [2]. Similarly, the International Atomic Energy Agency (IAEA) concluded as an important lesson from the accident in Fukushima that (quotation), 'The assessment of natural hazards needs to be sufficiently conservative'. [3].

Based on their quantitative decision criteria traditional probabilistic hazard assessment attempts to make a distinction between tolerated risks (that is, supposed to be infrequent 
events) and potentially more frequent risks that should be considered in the design. Therefore, these methods heavily rely on quantitative models and on data supporting their development. In the case of limited data support quantitative probabilistic assessments based on parametric models are very unreliable and may not lead to robust decisions $[4,5]$ even if complex uncertainty models are applied. Inspired by the well-known book of Nassim Taleb [6] an alternative distribution-free (and therefore parameter free) approach was developed to address the possibility of highly-improbable extreme natural hazards and the associated severe impact in the design and in the risk assessment of critical infrastructures. This approach is intended to complement traditional assessment methods supporting robust decision making. Accordingly, extreme events of natural origin are treated as 'black swan' events. They are very rare, but have an extreme impact. According to Taleb [6] such events belong to 'extremistan'. The design of critical infrastructures shall have sufficient safety margins or shall include specific safety features to prevent catastrophic consequences of such extremes as long as they cannot be excluded during their limited lifetime.

\section{METHOD DESCRIPTION}

The method proposed here makes use of some general properties of the mathematical theory of records and of the theory of heavy-tailed distributions. The method assumes that the recurrence of extreme natural events follows a stochastic process which is caused by the same, but unknown mechanism (corresponds mathematically to the i.i.d assumption for a sequence of random variables (rvs))). Additionally it is assumed that their magnitude characterizing their impact on civil or natural objects is following a heavy-tailed distribution. The latter is a direct consequence of the Fisher-Tippett theorem under the i.i.d. assumption; see Embrechts [7]. The validity of the assumptions can be expanded to the more general case of weak asymptotic convergence to a Homogeneous Poisson Process (HPP) (e.g. for Poissonian Random Measures (PRM)). This asymptotic convergence is proven for point processes of exceedances over a threshold using the Kallenberg theorem, Embrechts [7]. Note that any new record represents an exceedance over a threshold. It is frequently believed that the occurrence of extreme natural hazards can be explained as a phenomenon of self-organized criticality. The mathematical description of self-organized criticality directly leads to extreme value theory and heavy-tailed distributions, Woo [8]. Therefore, the mathematical prerequisites for the applicability of the assumptions of the method are less restrictive than the standard assumptions in probabilistic risk assessments (see for example section 2.2.2 in NRC [9]) and in traditional probabilistic hazard assessment.

2.1 General properties of the mathematical theory of records and of heavy-tailed distributions

A detailed overview on the mathematical basis of the theory of records and of heavy-tailed distributions can be found in Nevzorov [10], Cook and Nieboor [11] and in the mathematical text books of Embrechts [7] and Resnick [12]. Here we are interested in some general properties of the theory.

1. The expected number of records (denoted as $\mathrm{N}_{\mathrm{n}}$ ) in a sequence of $n$ observations is given by:

$$
E\left(N_{n}\right)=\sum_{j=1}^{n} \frac{1}{j} .
$$


2. The ratio between the largest and the second largest record in a record counting process for a heavy-tailed distribution converges asymptotically to a factor of 2 . The probability that the next record value will exceed the second largest (the previous) record value by a factor of 2 or more is equal to:

$$
\frac{2}{n}
$$

From these general properties, we can derive a set of interesting conclusions. The longer the historical period of observations the more reliably we can predict the magnitude of the next extreme record event. In other words, if we have a reasonably complete catalogue of events including for example observations of 100 registered extremes in the region of interest, the probability that the largest recorded value will be exceeded by a factor of 2 or more by a future event is just two per cent eqn (2). This conclusion can be expanded by estimating the probability of observing a new record value during the remaining (or planned) lifetime of a critical infrastructure. For this purpose, we estimate the number of record values as a function of the number of observations (counting discrete events) or of time (e.g. applicable for example to flood observations in terms of annual maxima).

Table 1 shows the expected number of records in function of the length of the observation records calculated from eqn (1).

From Table 1 it can be seen that the longer the time span that is covered by the registered observations the less likely it is that the observed largest event (the last record) will be exceeded during the residual (or planned) lifetime of a critical infrastructure. This can be illustrated by an example. Assume the planned lifetime of a critical infrastructure is 60 years (e.g. for a new NPP). Additionally, assume that the last record observation occurred just before the commissioning of the critical infrastructure of interest. Given an observation period of 83 years (e.g. for annual maxima of floods or another extreme weather condition) then the expected time till the next record is expected to be observed is 143 years. This is significantly longer than the planned lifetime of the infrastructure. The application of the method for seismic and tsunami data available before the 2011 disaster for the Japanese coast near the Fukushima plant would have allowed to predict an earthquake of a magnitude up to 9.5 with a subsequent huge tsunami (up to more than $30 \mathrm{~m}$ flood height). The probabilistic tsunami hazard analysis performed by TEPCO [13] related such extreme scenarios to the tolerable risk area.

Table 1: Expected number of records and time (or additional events) between records.

\begin{tabular}{lcc}
\hline $\begin{array}{l}\text { Number of observations } \\
\text { (e.g. annual maxima, or events) }\end{array}$ & $\begin{array}{c}\text { Expected Number } \\
\text { of records }\end{array}$ & $\begin{array}{c}\text { Time (or number of additional } \\
\text { events expected before record) } \\
\text { between subsequent records, [years] }\end{array}$ \\
\hline 11 & 3 & N.A. \\
31 & 4 & 20 \\
83 & 5 & 52 \\
226 & 6 & 143 \\
616 & 7 & 490 \\
\hline
\end{tabular}




\section{2 'Black swan' events}

The key element of the method proposed consists in the prediction of the largest extreme event to be expected during the residual or planned lifetime of a critical infrastructure. For many types of natural hazards, it can be assumed that the design performance goals for very rare extremes consist in the prevention of large consequential losses and not in a subsequent operation of the plant after the event. Once the plant will not be operated after the extreme event occurred, probabilistic hazard curves from traditional methods are not needed to be extrapolated beyond the scope of this extreme event. Under these conditions it is sufficient to design safety features to mitigate the consequences of the event while it is not required to maintain operability of the plant. For example, in the nuclear industry WENRA [2] formulated as the safety goal for such extremes that cliff-edge effects shall be avoided.

In accordance to this objective, we are interested in future events that are so strong that they will be considered a surprise to external observers. These are the properties of 'Black swan events'. For being a surprise, the event shall exceed all previously observed events. Thus, it represents a mathematical record. Furthermore, it shall exceed all previously observed events by a significant amount to be a surprise. Using the asymptotic properties of extremes with characteristics of a heavy-tailed distribution eqn (2) it is reasonable to assume that the magnitude of this next extreme record event exceeds the previously observed record value by a factor of 2 (it may be weaker). The probability of such a significant exceedance of the previous record in a region with a long period of historical registrations is rather small eqn (2). Certainly, such an extreme event will be considered as surprisingly strong and surprisingly rare.

In accordance with the analysis objective, the time period till this record event is expected to occur shall be larger than the lifetime of the infrastructure. If the data series available is too short to set a reliable design limit (e.g. the next predicted record event may occur during the residual lifetime of the plant) the method suggests predicting the magnitude of the second subsequent record event in the future sequence of records. Accordingly, this is an event that exceeds the magnitude of the largest recorded value in the data series by a factor of 4. Correspondingly, the waiting period till such an event will occur, is significantly longer eqn (1).

\subsection{Characterization of the magnitude of extreme events}

For the evaluation of the impact of extreme, highly-improbable events their magnitude shall be defined in a way that is related to engineering computations. Risk and design applications shall be aimed at preventing disasters, and disasters are associated with large scale damage. The process of damaging an object is a nonlinear process for which it is necessary to perform work. For performing work energy is needed. Therefore, the most appropriate parameter is energy and it is reasonable to use a measure for the intrinsic energy of the external event to characterize its ability to perform destructive work. It is worth to note that in semi-quantitative scales, this approach is general practice. In seismology, scientists use moment magnitude and intensities, for tornados the Fujita scale is related to wind speed and thus to energy, similarly for storms the Beaufort scale can be related to wind speed. In all cases, a measure of energy is linked to at least a semi-quantitative, sometimes descriptive measure of damage. For a 
more quantitative approach it is suggested to use the following characteristics for the characterization of extreme natural events:

- Seismology - Arias intensity, Klügel [14]

- High Wind - kinetic energy

- Extreme river flows - kinetic energy

- High Temperature - rate of radiation energy transmitted from sun on an object based on the Stefan-Boltzmann equation.

$$
Q=\varepsilon \sigma\left(T_{\text {air }}^{4}-T_{o b j e c t}^{4}\right)
$$

Here, $T_{\text {air }}$ is the ambient air temperature, $T_{\text {object }}$ the average surface temperature, $\sigma$ is Stefan's constant and $\varepsilon$ is the emissivity of a grey body, representing the object.

To obtain standardized conditions and for avoiding the need to solve a radiation heat transfer problem, we assume an average surface temperature of the object to be $303.15 \mathrm{~K}$. For central European conditions, this corresponds to a warm summer day. Under current central European conditions, the design of critical infrastructures certainly matches this design temperature even over a period of 24 hours of operation. By doubling the radiation energy for the next record event, relating it to the previous record value and solving for the ambient air temperature of the new (next) record event we obtain eqn (4):

$$
T_{\text {new }}=\left[\left(2\left(T_{\text {old }}^{4}-T_{\text {object }}^{4}\right)=T_{\text {object }}^{4}\right)\right]^{0.25} .
$$

A principal alternative parameter which is based entirely on characteristics of the hazard is the total radiation heat transmitted to an object over a mission time MT:

$$
Q_{R}=\int_{0}^{M T} \varepsilon \sigma T_{a i r}^{4} d t .
$$

This parameter can be applied for more general applications, e.g. outside the range of European conditions.

\section{PRACTICAL APPLICATION}

The method developed was applied for the NPP Goesgen for different types of natural hazards, Klügel [14]. Here, we apply the method for the load cases 'High Wind' and 'High Temperature'.

\subsection{High wind}

Due to modifications of the measurement installations NPP Goesgen possesses harmonized wind speed data only for a 28 -year measurement period. During this period, four records were observed that are presented in Table 2 . The observed number of records corresponds to the expectations for an i.i.d. recurrence process (Table 1).

The next record value yields from the multiplication of the observed largest wind speed by the factor square root of 2 (doubling the kinetic energy under the assumption of constant density). Therefore, the prediction of the next record results in a wind speed value of $44.7 \mathrm{~m} / \mathrm{s}$. 
Table 2: Wind speed record values at NPP Goesgen site (10 min average values).

\begin{tabular}{cc}
\hline Year & Wind speed, $[\mathrm{m} / \mathrm{s}]$ \\
\hline 1987 & 27.6 \\
1990 & 28.7 \\
1991 & 29.0 \\
1999 & 31.6 \\
\hline
\end{tabular}

The NPP Goesgen plans to operate till around 2040. Based on Table 1 for a number of observations of 28 (with 16 years already passed) we have to expect that the predicted next record value may occur during the residual lifetime of the plant. As a result, it is more reasonable to consider the second in the sequence of records value for the safety review and for the design of mitigating safety features.

According to the method proposed, this record value corresponds to a wind speed of 63.2 $\mathrm{m} / \mathrm{s}$. Based on the data in Table 1, we can conclude that this 'black swan' event is expected to occur after the final decommissioning of the plant. The probability that the second in the sequence record will be equal or higher than this value is 0.004 . Using the predicted second record value for approval of the design of the safety functions of the NPP leads to a conservative safety directed approach. For NPP Goesgen, this value is within the design values.

In Switzerland, the design hazard usually is defined as the mean wind speed for a frequency of exceedance of $10^{-4}$ per year. The detailed construction of a hazard curve using standard methods like extreme value analysis (using the GEV distribution both stationary and time-dependent models) and logic trees (for combination of alternative models) results in a design wind speed of $43.2 \mathrm{~m} / \mathrm{s}$. The mean is understood here as the mean of the joint aleatory and epistemic uncertainty distributions.

This difference between the 'black swan' extreme events and the usual design approach has important risk implications. The development of traditional hazard curves for extreme events of natural origin based on a limited set of observation data in conjunction may lead to a significant underestimation of risk for critical infrastructures. To achieve the objective of performing a robust hazard assessment in compliance with the requirements of the IAEA [3] additional considerations like the method proposed may be of significant benefit to avoid overly optimistic results.

\subsection{High temperature}

High temperatures usually do not directly affect the integrity of structures and components. They may affect the functional performance of equipment. The spreading use of digital I\&C may make many installations more vulnerable to environmental effects. In the nuclear industry high temperatures may affect the capacity of the plant for residual heat removal in case of a loss of offsite power accident. For the NPP Goesgen site, a limited set of observations is available. The reason is again the modification of the measurement system that does not allow for a technically qualified homogenization of data. Data are available from 2000 to 2015. According to the theory of records (compare Table 1) approximately three record values are expected in the data set. Indeed, three record values were observed. Therefore, 
Table 3: Record air temperatures observed at NPP Goesgen.

\begin{tabular}{lc}
\hline Year & Maximum air temperature, $\left[{ }^{\circ} \mathrm{C}\right]$ \\
\hline 2000 & 32.3 \\
2002 & 34.7 \\
2003 & 36.5 \\
\hline
\end{tabular}

the i.i.d assumption cannot be rejected for the data set. The observed record values are shown below in Table 3 .

Using the Stefan-Boltzmann equation, we predict the next record value by multiplying the largest observed value by a factor corresponding to eqn (4). This results in a predicted record temperature of $42.6^{\circ} \mathrm{C}$. This record value was used for the safety review of the plant. Due to the short observation period, it would be worth to evaluate the next record value, too. Because high temperature conditions cannot directly cause a plant transient due to the design of NPP Goesgen (several independent cooling water systems) another way was selected. For the current program of safety upgrades for NPP Goesgen, the design temperature was increased to $45^{\circ} \mathrm{C}$. This ensures additional safety margin for high temperature conditions.

It is worth to mention that the peak design air temperature derived from a statistical study for a frequency of exceedance of $10^{-4} / \mathrm{a}$ is $40.1^{\circ} \mathrm{C}$. Again, it cannot be fully excluded that risk studies that are based on hazard curves derived from a limited set of data may not be conservative.

\section{CONCLUSIONS}

A new method for the hazard assessment of extreme natural events based on Black Swan Theory was developed. The method was applied with reasonable results for the safety review of NPP Goesgen with respect to extreme events of natural origin (see also Klügel, [14]). The comparison of the results with traditional statistical methods that are frequently used in probabilistic hazard and in risk assessments for critical infrastructures, indicates, that traditional methods may not be sufficiently robust especially in the case of limited observational data sets. The latter is frequently the case. The new method can be recommended as a supplementary tool for plausibility checks to prove the robustness of results from complex risk studies or in the process of site evaluation for critical infrastructures.

\section{REFERENCES}

[1] Klügel, J.U., Lessons not yet learned from the Fukushima disaster. Acta Geodaetica Geophysica, 50, pp. 5-19, 2015. http://dx.doi.org/10.1007/s40328-014-0084-2

[2] WENRA, "Guidance document issue T: natural hazards head document." guidance for the WENRA safety reference levels for natural hazards introduced as lesson learned from TEPCO Fukushima dai-ichi accident, 2015.

[3] The Fukushima Daichii Accident, report by the director general, IAEA, Vienna, 2015.

[4] Klügel, J.-U., Uncertainty analysis and expert judgment in seismic hazard analysis. Pure and Applied Geophysics, 168, pp. 27-53, 2011. http://dx.doi.org/10.1007/s00024-010-0155-4

[5] Klügel, J.-U., Error inflation in probabilistic seismic hazard analysis. Engineering Geology, 90, pp. 186-192, 2007. 
http://dx.doi.org/10.1016/j.enggeo.2007.01.003

[6] Taleb, N., The black swan, the impact of the highly improbable, Random House, 2007.

[7] Embrechts, P., Klüppelberg, C. \& Mikosch, T., Modelling Extremal Events for Insurance and Finance, Springer: New York, Berlin, Heidelberg, 1997.

[8] Woo, G., The Mathematics of Natural Catastrophes, Imperial College Press, London, 1999.

[9] NRC, Handbook of parameter estimation for probabilistic risk assessment, NUREG/ CR-6823, Washington: U.S. Nuclear Regulatory Commission, 2003.

[10] Nevzorov, V.B., Records: mathematical theory, translations of mathematical monographs. American Mathematical Society, 194, 2001.

[11] Cook, R.M. \& Nieboor, D., Heavy-Tailed Distributions: Data, Diagnostics and New Developments, RFF DP 11-19, Resources for the Future, Washington, 2011.

[12] S. F. Resnick, Heavy-Tail Phenomena. Probabilistic and Statistical Modeling., Berlin, Heidelberg, New York: Springer, 2009.

[13] Annaka, T., Satake, K., Sakakiyama, T., Yanagisawa, K. \& Shuto, N., Logic-tree Approach for Probabilistic Tsunami Hazard Analysis and its Applications to the Japanese Coasts. Pure and Applied Geophysics, 164, pp. 577-592, 2007. http://dx.doi.org/10.1007/s00024-006-0174-3

[14] Klügel, J.-U., Consideration of "Black Swan" Events in the Seismic Safety Review and the Seismic Upgrade Programme of Existing Nuclear Power Plants - the NPP Goesgen Example, in Post-SmiRT23 Seminar, Istanbul, 2015. 